taktkategorie. Nach Böltken ist das „normale“ Nachbarschaftsverhältnis zwischen Türken und Deutschen zwar relativ konfliktfrei, doch deutlich distanziert.

Die Unterschiede in der Kontakthäufigkeit und -intensität betreffen nach Geissler (1992: 160) auch die jüngere Generation mit türkischem Migrationshintergrund.

Von Wichtigkeit im Kontext des wohnnachbarschaftlichen Kontakt- und Konfliktgeschehens scheint der Faktor der Sichtbarkeit und das damit vielfach einhergehende Ausmaß an sozialer Distanz zu bestimmten Gruppen zu sein. Wir haben dazu auch in unsere Befragung eine Frage eingeschaltet, die wir aus dem deutschen ALLBUS übernahmen, aber im Wortlaut und bezüglich der zu bewertenden Migrantengruppen modifizierten. Die Bedeutsamkeit des Faktors der sozialen Distanz wurde bereits in zahlreichen Studien herausgearbeitet, von denen hier aber bloß einige angeführt werden sollen (Institut für Demoskopie Allensbach 1982; Just \& Mülhens 1982; Drevermann et al. 1983; Kuhlbrodt 1984; Allerbeck \& Hoag 1986; Schubarth 1990; Plasser \& Ulram 1991; Eckert \& Kissler 1992; Fischer 1992; Markefka 1995; IHS 1998).

\title{
4 Sozialkontakte und Konflikte im Kontext der Erforschung von Nachbarschaftsbeziehungen
}

\subsection{Die Kontakthypothese}

Die vorliegenden empirischen Befunde weisen zwar in keine einheitliche Richtung, sie sind aber als Rechtfertigung ausreichend, um davon auszugehen, dass die konkreten Erfahrungen, die Inländer mit Migranten in ihrem unmittelbaren Wohnumfeld machen, einen Einfluss auf die Einstellungen der betreffenden Personen gegenüber Zuwanderern allgemein bewirken können. Es ist damit also die als theoretische Ausgangshypothese in zahlreiche sozialwissenschaftliche Studien einfließende Kontakthypothese angesprochen.

Die Pionierstudie, welche viele künftige Untersuchungen bis zum heutigen Tage wesentlich beeinflusste, ist G. W. Allports „The Nature of Prejudice“ (1954, 1956). Diese bezieht sich zwar nicht primär auf nachbarschaftliche Interaktionen, deckt diese aber mit ihrem Fokus auf interethnischen Kontakten ebenfalls ab. Allport ging von einer präzisen Taxonomie von Faktoren aus, die die Resultate interethnischer Kontakte determinieren. Zu diesen Faktoren zählen (a) der quantitative Aspekt des Kontakts (z.B. Häufigkeit, Dauer, Zahl der involvierten Personen, Vielfalt), der (b) Status des Kontakts (abhängig davon, ob die Angehörigen der darin involvierten Minorität den gleichen, einen unter- oder übergeordneten Sozialstatus innehaben), die (c) Rollenaspekte des Kontakts (kompetitiver oder kooperative Sozialbeziehung, Unterordnung), (d) die soziale Atmosphäre, in welche der Sozialkontakt eingebettet ist (findet der Kontakt freiwillig oder unfreiwillig statt, ist er „,natürlich“ oder „künstlich“, typisch oder stellt er eine Ausnahmesituation dar), (e) die Persönlichkeitsmerkmale (Alter, Bildungsniveau, Vorurteilslevel) der in den Sozialkontakt involvierten Individuen 
sowie den (f) Ort, an welchem der Kontakt stattfindet (zufällig, Wohnbereich, Berufswelt etc.) (vgl. dazu ebenda: 262 f.).

Die grundlegende Annahme im Zusammenhang mit der Einbringung der Kontakthypothese in die mikrosoziologische Wohnnachbarschaftsforschung ist:

Kontakte auf Basis der räumlichen Nähe der Wohnstandorte führen

1) zu Kontakten der Bewohner,

2) zu einem Mehr an Toleranz und zur Reduktion von Intergruppenvorurteilen unter den Nachbarn,

3) unter besonders optimalen Bedingungen nicht nur zur Modifikation von Einstellungen, sondern sogar zur Veränderung von Verhaltensmustern in allen beteiligten Gruppen.

Die Kontakthypothese ,, besagt, dass Kontakte - und besonders solche unter Statusgleichen - der Entstehung und Stabilisierung von Vorurteilen entgegenwirken bzw. ihre Reduktion begünstigen " (Hill 1984: 364). Selbstverständlich war es auch Allport bewusst, dass die von ihm angegebenen Voraussetzungen eine idealtypische Konstellation repräsentieren, die in der sozialen Realität wohl kaum anzutreffen sein dürfte (Scandella 2003: 7; vgl. auch Hewstone \& Brown 1986: 4). Tatsächlich sind daher Studien auf der Basis von Allports Konzept zu recht kontradiktorischen Resultaten gelangt (Scandella 2003: 7).

Das Zentrum für Türkeistudien (1995: 136) beispielsweise sowie Silbermann \& Hüsers (1995) kamen in ihren Untersuchungen zu dem Resultat, dass in je mehr Bereichen und je öfter Befragte Kontakt zu Ausländern hatten, sich diese umso weniger fremdenfeindlich äußerten. Empirische Untersuchungen an westdeutschen Jugendlichen, die häufigen Umgang mit ausländischen Schul-/Berufskollegen hatten, bestätigten den Einfluss, den der Kontakt mit Migranten auf Toleranz hat (Hessler 1993: 154 f.; Hess 1996: 247). Eine Bestätigung der Kontakthypothese erbrachten auch die Untersuchungen von Zeul \& Humphrey (1971), Cagle (1973) und Schwinges $(1979,1982)$.

Eine wichtige Kritik an der Kontakthypothese bezieht sich darauf, dass es ihr an Klarheit bezüglich der analytischen Bezugsebene mangelt (Pettigrew in Hewstone \& Brown 1986: 179 ff.). Viele Arbeiten, die auf der Kontakthypothese basieren, geben vor, sich auf die Intergruppenebene zu beziehen. Faktisch untersuchen sie jedoch interpersonelle Kontakte (Doise in Moscovici 1984: 263). Das Grundproblem besteht darin, dass die kausalen Zusammenhänge zwischen Stereotypen und Interaktionen nicht wirklich klar sind. Viele Autoren (vgl. Hewstone \& Brown 1986: 16) gehen davon aus, dass zwischen Stereotypen und Kontakten eine dynamische Beziehung wechselseitiger Kausalität besteht.

Weiters hat sich immer wieder gezeigt, dass Kontakt keinen ausreichenden Faktor für eine Verbesserung der Intergruppenbeziehungen darstellt und diese sogar verschlechtern kann (Scandella 2003: 8).

Kontakt scheint vielmehr nur unter bestimmten optimalen Bedingungen in der Lage zu sein, Beziehungen zwischen einander ablehnend gesinnten Personen zu verbessern (Cook 1962; Pettigrew 1971, 1986; Amir 1976). Vielfach wurde nachge- 
wiesen, dass es weniger die Kontaktfrequenz, sondern vielmehr die Art der Interaktionen ist, welche für positive Veränderungen ausschlaggebend ist (Amir 1969, 1976). Bloße räumliche Nähe schafft noch keine intensiven Kontakte, denn diese werden üblicherweise selektiv eingegangen (Gans 1974: 189; Friedrichs 1977: 264; Soen \& Agnon 1980: 276). Es sind auch Befunde vorhanden, dass sich gerade durch Kontakt Vorurteile sogar noch verfestigen können (vgl. Slavin 1983; Stephan 1987; Hewstone \& Brown 1986; Omoto \& Borgida 1988).

Kritiker der Kontaktyhpothese (Amir 1969: 338 ff.; Dangschat 1997) wiesen darauf hin, dass Kontakte in unteren sozialen Ebenen der Gesellschaft viel eher zu Konflikten und Ausgrenzungen als zur Integration führen. Kontakt schafft Sympathie bei Gebildeten und/oder Mittelschichtangehörigen, nicht aber bei marginalisierten Inländern den Migranten gegenüber. ${ }^{17}$ Schon Zeul \& Humphrey (1971) zweifelten die Verallgemeinerbarkeit der Kontakthypothese an und betonten die Statusabhängigkeit von Einstellungen.

Eine entscheidende Frage ist also, unter welchen typischen Bedingungen Kontakte als angenehm oder unangenehm empfunden werden, denn Kontakte zwischen unterschiedlichen Ethnien neigen häufig ohnehin per se dazu, unangenehm und spannungsreich zu sein (Flohr 1994: 247).

Die Kontaktbereitschaft variiert ebenso wie die Vorurteilsbereitschaft auf Seiten der Majorität (der Inländer) mit dem Alter (je älter, desto vorurteiliger) und der Schulbildung (je höher das Bildungsniveau desto geringer die Vorurteile) (vgl. Friedrichs 1990: 317).

Die Bedeutung des Kontaktes für den Abbau von Vorurteilen (sog. Homan'sche Regel - „Kontakt schafft Sympathie“) ist also vor dem Hintergrund der bislang geleisteten Forschung nicht eindeutig festzulegen (Dollase 1994: 421). Die Resultate der empirischen Untersuchungen sind äußerst uneinheitlich, teilweise sogar kontradiktorisch (vgl. Brewer \& Kramer 1985: 233 f.). Reuband (1989: 273) vermutet eine Interdependenz der beiden Variablen Häufigkeit und Art der Kontakte, das heißt, freundschaftliche Kontakte vermindern einerseits negative Einstellungen und gleichzeitig begünstigen reduzierte Vorurteile die Herausbildung freundschaftlicher Beziehungen. Die Variable „Häufigkeit von Kontakten mit Ausländern“ „, ist an sich wenig aussagekräftig“ (Jäger 1995: 62), denn diese variiert ganz entscheidend mit der Variable „Art der Kontakte“. Welches Gewicht dabei den Nachbarschaftskontakten zukommt, ist nicht deutlich herausgearbeitet worden bzw. bestehen darüber konträre Befunde. Gehring \& Böltken (1985), die sich mit der Kontaktproblematik intensiv auseinandergesetzt haben, weisen den nachbarschaftlichen Kontakten eine inferiore Rolle gegenüber den intensiveren Freundschafts- und familiären Kontakten zu.

17 Diskussionsbeitrag von J. S. Dangschat anlässlich des Vortrags von H. Häussermann „Zuwanderung und Stadtentwicklung“" am 29.4.1997, Wien, BMWV sowie Vortrag am 9.6. 1998 anläßlich der Tagung „Migration and Sustainable Urban Development“ in Wien. 
Der Forschungsstand macht klar, dass sowohl das Zustandekommen als auch die Auswirkungen interethnischer Kontakte von einer Vielzahl von Determinanten abhängen und nicht auf eine simple Kausalität zwischen der räumlichen Nähe der Wohnstandorte und der Kontakthäufigkeit reduziert werden können.

\subsection{Konflikte}

„Soziale Beziehungen sind immer auch potentielle Konfliktfelder" (Schulz zur Wiesch 1982: 271). Unter sozialen Konflikten verstanden die Konflikttheoretiker ,alle strukturell erzeugten Gegensatzbeziehungen von Normen und Erwartungen, Institutionen und Gruppen “ (Kiss 1977: 218).

Die mediale Berichterstattung über interethnische Nachbarschaftsbeziehungen konzentrierte sich bislang vor allem auf das Konfliktpotential des Tür-an-TürWohnens von Inländern und Migranten (vgl. Besser Wohnen 11, Oktober 1992: 18 f.), das zwar ein Nebeneinander, aber kein Miteinander sei (Der Standard, 12./13. 10.1991: 8). Vor allem für die Wiener Gemeindebauten wurden erhebliche Spannungen zwischen Inländern und „Neoösterreichern“ attestiert (Menasse 1996; Tanzer 1996; Der Standard, 10.11.1998: 36; Lohmeyer \& Wetz 2005). Es wurde angesichts des Faktums, dass es sich bei einem Drittel bis zur Hälfte (genaue Zahlen existieren nicht) der 13.000 Vorgemerkten für wiedervermietete Wiener Gemeindewohnungen um inzwischen eingebürgerte Zuwanderer handelte (Der Standard, 10.11.1998: 9), auch von einer künftigen Verstärkung dieser Konflikte in Wien ausgegangen. Vieles an diesen Konfliktszenarien war bislang allerdings bloße Spekulation, denn empirisch fundierte Ergebnisse waren für Wien nur wenige ${ }^{18}$ (z.B. Reppé \& Reiter 1997; Kohlbacher 2000; Ludl 2003) vorhanden.

Nach Heitmeyer (1994: 384) ist davon auszugehen, dass jede ,Gesellschaft mit unterschiedlicher ethnischer Zusammensetzung eine konflikthafte Gesellschaft ist". Kontakt und Konflikt stehen in einem unmittelbaren Dependenzverhältnis zueinander. Kontakt kann Sympathie schaffen, kann jedoch Konflikte auch erst erzeugen. Konflikt ist ohne Kontakt unmöglich. Der Kulturkonflikt erfordert nach Heintz (1968: 119) und Heitmeyer (1994: 385) intensiven Kontakt zwischen zwei Gruppen; weiters die Unvereinbarkeit von Werten und die Nichtbereitschaft, die Überlegenheit fremder Werte anzuerkennen.

Die Kollision divergierender Verhaltensmuster stellt eine Hauptursache für Konflikte im Wohnbereich dar. Das Wohnverhalten vieler Migranten ist durch die Sozialisation in ein anderes Wertesystem und ein anderes räumliches Umfeld (oft ländlicher Raum) als im Aufnahmeland geprägt.

18 Vgl. dazu auch die Dokumentation zur Vortragsveranstaltung „Gemeindebau im Gespräch - Entwicklungen und Perspektiven im kommunalen Wohnbau in Wien“ (http://www.gerda.univie.ac.at/ifs/files/workshop/gemeindebau.pdf?PHPSESSID=2b98a139ed6c668394fcf $8 \mathrm{c} 52 \mathrm{bd} 070 \mathrm{c} 6)$. 